\title{
Il ruolo della biopsia renale nella malattia di Anderson-Fabry
}

\author{
Sandro Feriozzi, Mario Mangeri \\ Nefrologia e Dialisi, Ospedale Belcolle, Viterbo
}

\begin{abstract}
Renal biopsy in Anderson-Fabry nephropathy
There are two major fields in which renal biopsy in Anderson-Fabry (AF) disease may play a role. The first is investigation, when we are involved in a trial to investigate particular aspects of AF nephropathy or the effect of therapy. In this case, the reason and the time to perform a renal biopsy are planned by the scientific board. The second is when we are looking after a patient with AF with renal involvement. In this case, in the typically affected male, biopsy can be useful but is not mandatory. Instead, in an asymptomatic female with only mild proteinuria, or a male adult over 40 years of age with normal renal function, it is important to perform a renal biopsy. Indeed, it will possible to demonstrate organ involvement of AF disease, and so it will be correct to start enzyme replacement therapy. Moreover, sometimes a patient may have a clinical picture not immediately ascribable to AF disease (macrohematuria, nephrotic proteinuria, etc.); in these cases histological examination may reveal a double pathology (IgA nephropathy, diabetic changes, etc.) associated with typical changes of Fabry nephropathy.
\end{abstract}

Keywords: Fabry nephropathy, Proteinuria, Renal biopsy, Renal function

La mutazione del gene che sintetizza per l'enzima agalsidasi alfa determina una riduzione o mancanza dell'enzima con conseguente deposizione intracellulare di prodotti del metabolismo dei glicosfingolipidi non correttamente degradati (Gb3). II gene è situato sul cromosoma $X$ quindi la patologia sarà manifesta pienamente nei maschi e più tardiva e con un decorso meno aggressivo nelle femmine (1).

Il coinvolgimento renale è in grado di determinare in modo significativo la prognosi della patologia, pertanto porre tempestivamente la diagnosi di nefropatia in corso di malattia di Anderson-Fabry (AF) è un elemento decisivo per il decorso clinico.

La nefropatia di AF si manifesta nei casi classici (maschi) con modesta proteinuria nella seconda e terza decade, con proteinuria e riduzione del filtrato glomerulare associato o meno a ipertensione arteriosa, e nella quarta decade con un quadro conclamato di insufficienza renale (2).

Accepted: March 13, 2017

Published online: July 28, 2017

Indirizzo per la corrispondenza:

Prof. Sandro Feriozzi

UOC Nefrologia e Dialisi

Ospedale Belcolle

Strada Sammartinese snc

01100 Viterbo

sandro.feriozzi@asl.vt.it
Le lesioni istologiche che si possono osservare sono dovute alla deposizione intracellulare di Gb3 che è in grado di attivare una serie di meccanismi locali che provocano attivazione dell'immunità innata, della flogosi e successivamente determinano la fibrosi e la sclerosi del tessuto (3).

Alla microscopia ottica la deposizione di Gb3 si osserva nel glomerulo (podociti ed endotelio) (Fig. 1), nella parete dei vasi delle arteriole e nell'epitelio dei tubuli distali. L'immagine che si osserva è quella di una vacuolizzazione del citoplasma poiché i solventi lipidici utilizzati per le colorazioni standard dissolvono i lipidi (aspetto a nido d'ape). Nei preparati per la microscopia elettronica per includere i tessuti si utilizzano resine, pertanto il Gb3 viene fissato e compare come un corpuscolo all'interno delle cellule. Nei preparati per la microscopia elettronica questi corpuscoli assumono l'aspetto di inclusi con lamelle concentriche e sono chiamati corpi zebrati (4).

Per valutare il valore delle informazioni derivabili da una biopsia renale occorre distinguere se siamo nell'ambito di uno studio multicentrico della nefropatia o se ricerchiamo informazioni cliniche per il nostro paziente. Nel primo caso sarà utile aderire alle indicazioni del protocollo in atto ed eseguire la biopsia. I dati ottenuti potranno essere condivisi con altri gruppi e la numerosità del campione permetterà un miglioramento delle nostre conoscenze (5).

Da un punto di vista strettamente clinico dobbiamo suddividere le indicazioni nei seguenti contesti: maschi con forma classica, femmine nei quadri atipici (late-onset e sospetto doppia patologia) (Tab. I). 


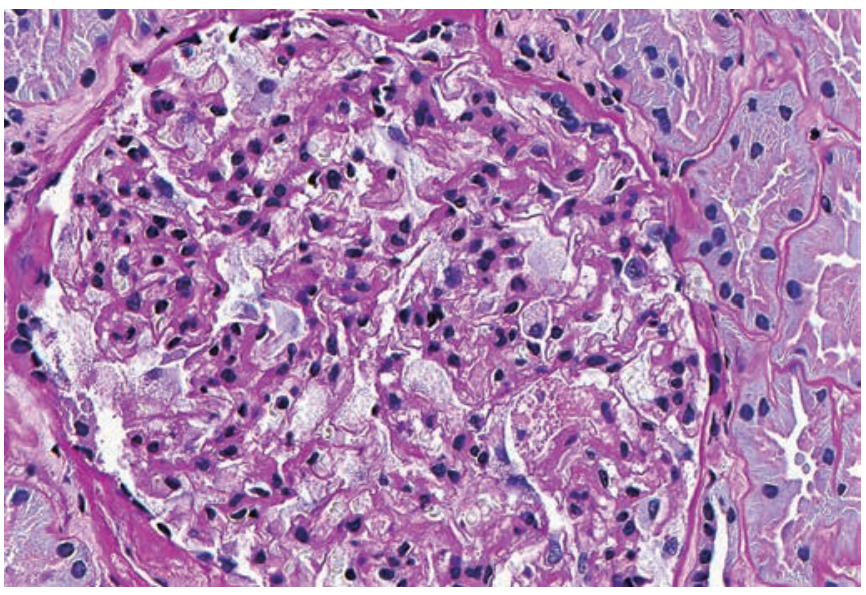

Fig. 1 - Microscopia ottica: alcune anse capillari in cui si osserva il citoplasma di alcuni podociti viscerali con la tipica vacuolizzazione (PAS 40x).

Nei maschi con decorso tipico iniziato in età pediatrica l'esecuzione della biopsia può essere utile ma certamente non è obbligatoria. I quadri istologici associati a proteinuria e riduzione del filtrato glomerulare sono noti e difficilmente condizioneranno le scelte terapeutiche (6).

Nelle donne la comparsa di una proteinuria seppur modesta (300-500 mg/24h) è indicazione alla biopsia, dopo aver ovviamente escluso altre patologie (infezioni urinarie ecc.). In questo caso infatti la dimostrazione della deposizione di Gb3 permette la diagnosi di coinvolgimento d'organo e costituisce elemento decisivo per l'inizio della terapia enzimatica sostitutiva. Questo approccio può essere utilizzato anche nei casi pediatrici quando non vi è una diagnosi certa (6-8).

In soggetti maschi adulti vi sono alcune forme di malattia che, per ragioni ancora non completamente chiarite, tendono a comparire tardivamente dopo i cinquant'anni e si caratterizzano per il prevalente coinvolgimento di un organo (cuore, rene). In questi casi la biopsia è importante per la dimostrazione della nefropatia e l'approccio terapeutico (9).

Vi sono poi condizioni nelle quali in un paziente sospettato di essere affetto dalla malattia compaiono segni nefrologici inusuali come una proteinuria nel range nefrosico o episodi

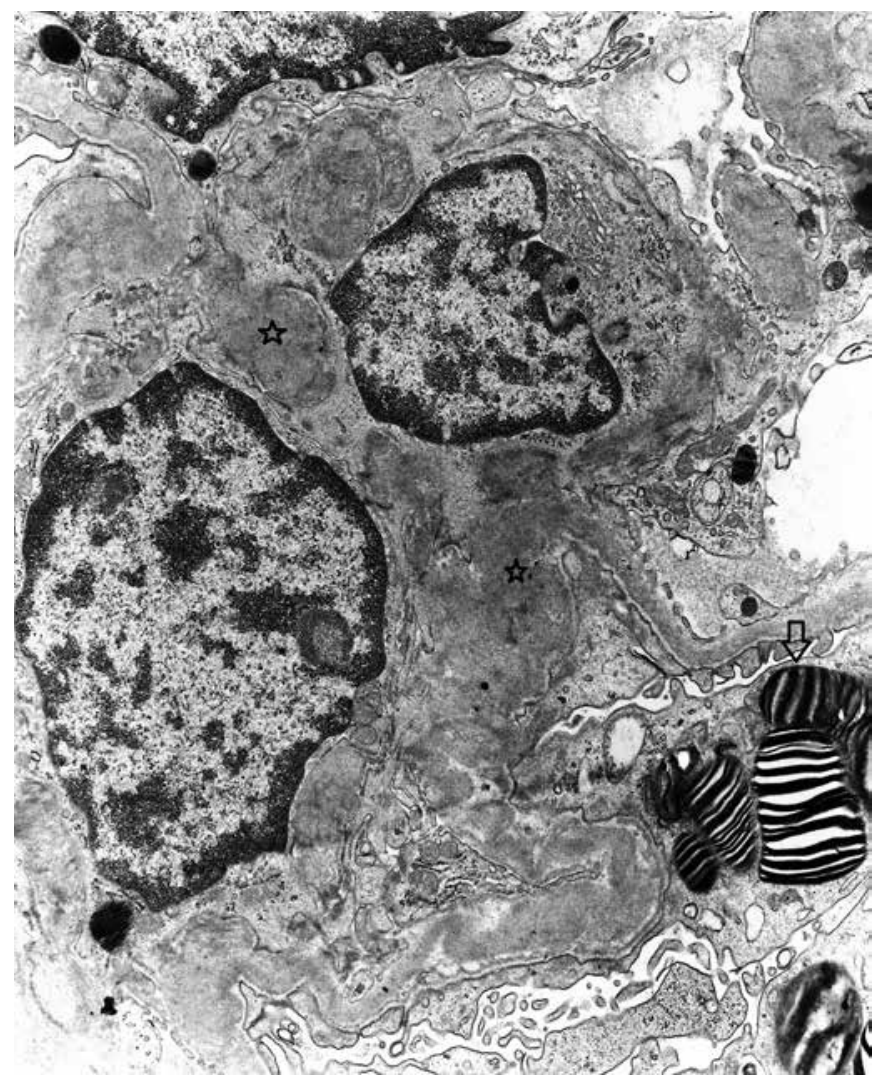

Fig. 2 - Microscopia elettronica: coesistenza di nefropatia IgA e nefropatia di Fabry; in area mesangiale si osservano deposti di immunocomplessi elettrondensi (stella), mentre nell'endotelio vi sono le tipiche inclusioni "corpi zebrati" (freccia). I depositi immuni erano positivi per IgA all'immunofluorescenza. (Per gentile permesso del Prof. Andrea Onetti Muda, Università Campus Bio-Medico, Roma) (UrPb 8000x).

di macroematuria. In questi casi l'esecuzione della biopsia è essenziale poiché sono stati descritti in letteratura quadri di doppia patologia (diabete, nefropatia IgA, vasculiti, Lupus ecc.) (6) (Fig. 2).

Ultimo punto è il ruolo che può avere una ripetizione della biopsia per valutare la terapia. È bene ricordare che in una biopsia renale sono presenti mediamente da 10 a 30 glome-

TABELLA I - Indicazioni alla biopsia renale nella malattia di Anderson-Fabry

\begin{tabular}{|c|c|c|}
\hline Clinica & Indicazione & Commento \\
\hline $\begin{array}{l}\text { Maschio con proteinuria modesta e riduzione della } \\
\text { funzione renale }\end{array}$ & Utile ma non indispensabile & Difficilmente le lesioni modificheranno la terapia \\
\hline Adulti maschi con sospetto di nefropatia & Assoluta & Diagnosi di late-onset \\
\hline $\begin{array}{l}\text { Quadri con segni atipici (proteinuria nefrosica, } \\
\text { macroematuria) }\end{array}$ & Assoluta & $\begin{array}{l}\text { Doppia patologia diabete, nefropatia IgA, Lupus, } \\
\text { vasculiti }\end{array}$ \\
\hline
\end{tabular}


ruli rispetto al patrimonio nefronico di 1.000 .000 per ciascun rene. Ne deriva che nel singolo caso è veramente difficile dedurre dalle lesioni notizie valide per una valutazione clinica (10). Completamente diverso è se la nostra biopsia, come detto prima, fa parte di un protocollo cui afferiscono altri gruppi. Una lettura centralizzata e condivisa tra i partecipanti allo studio potrà fornire elementi utili alla conoscenza della malattia (5). D'altra parte, stante la rarità della patologia, è praticamente impossibile che un singolo Centro possa svolgere questo tipo di studio da solo. Per i nefrologi il modello resta lo studio (10) nel quale sono stati valutati più di mille casi di nefropatia IgA raccolti in tutto il mondo e che ha permesso di mettere alcune certezze alle correlazioni clinico-istologiche della nefropatia IgA.

\section{Disclosures}

Financial support: No financial support was received for this submission.

Conflict of interest: The authors have no conflict of interest.

\section{Bibliografia}

1. Germain DP. Fabry disease. Orphanet J Rare Dis. 2010;22:5:30.

2. Branton $M H$, Schiffmann R, Sabnis SG, et al. Natural history of Fabry renal disease: influence of alpha-galactosidase $A$ activity and genetic mutations on clinical course. Medicine (Baltimore). 2002;81(2):122-38

3. Trimarchi $\mathrm{H}$. The kidney in Fabry disease: more than mere sphingolipids overload. Jornual of Inborn Errors of Metabolism and Screening. 2016;4:1-6.

4. Faraggiana T, Churg J, Grishman E, et al. Light-and electronmicroscopic histochemisty of Fabry's disease. Am J Pathol. 1981;103(2):247-62.

5. Fogo $A B$, Bostad L, Svarstad E, et al. All members of the International Study Group of Fabry Nephropathy (ISGFN). Scoring system for renal pathology in Fabry disease: report of the International Study Group of Fabry Nephropathy (ISGFN). Nephrol Dial Tansplant. 2010;25(7):2168-77.

6. Waldek S, Feriozzi S. Fabry nephropathy: a review - how can we optimize the management of Fabry nephropathy? BMC Nephrol. 2014;15:72.

7. Parini R, Feriozzi S. Females and children with AndersonFabry disease: diagnosis,monitoring, benefits of enzyme replacement therapy (ERT) and consideration on timing of starting ERT. Expert Opinion in Orphan Drug. 2013;1: 315-30.

8. Tøndel C, Bostad L, Hirth A, Svarstad E. Renal biopsy findings in children and adolescent with Fabry disease and minimal albuminuria. Am J Kidney Dis. 2008;51:767-76.

9. Niemann $M$, Rolfs $A$, Störk $S$, et al. Gene mutations versus clinically relevant phenotypes: Lyso-Gb3 defines Fabry disease. Circ Cardioasc Genet. 2014;7(1):8-16.

10. Corwin HL, Schwartz MM, Lewis EJ. The importance of sample size in the interpretation of the renal biopsy. Am J Nephrol. 1988;8(2):85-9. 